\title{
Recurrent patellofemoral instability rates after MPFL reconstruction techniques are in the range of instability rates after other soft tissue realignment techniques
}

\author{
Olivier E. Wilkens ${ }^{1} \cdot$ Gerjon Hannink ${ }^{2}$ (D) . Sebastiaan A. W. van de Groes ${ }^{1}$
}

Received: 9 March 2019 / Accepted: 29 July 2019 / Published online: 7 August 2019

(c) The Author(s) 2019

\begin{abstract}
Purpose Recurrent patellofemoral instability is a common knee injury in skeletally immature patients. Many surgical techniques have been described in the literature, all with different success rates. Purpose of this study was to perform a systematic review and meta-analysis of the available literature to assess recurrent patellofemoral instability rates after surgical treatment using MPFL reconstruction techniques and other soft tissue realignment techniques in skeletally immature patients.

Methods PubMed, Embase, Web of Science, and The Cochrane Library were searched to identify all original articles concerning the surgical treatment for patellofemoral instability in skeletally immature patients and that reported post-operative recurrent patellofemoral instability rates. Subsequently a risk of bias assessment was conducted and a meta-analysis was performed on reported post-operative recurrent patellofemoral instability rates after MPFL reconstruction techniques and other soft tissue realignment techniques.

Results Of the 21 eligible studies (448 knees in 389 patients), 10 studies reported on MPFL reconstruction techniques using different grafts and fixation techniques and 11 reported on other soft tissue realignment procedures. In total, 62 of the 448 (13.8\%) treated knees showed recurrent patellofemoral instability during follow-up. The overall pooled recurrent patellofemoral instability rate was estimated to be 0.08 (95\% CI 0.02-0.16). For MPFL reconstruction techniques, the pooled recurrent patellofemoral instability rate was estimated to be 0.02 (95\% CI 0.00-0.09). For the other soft tissue realignment techniques, the pooled rate was estimated to be 0.15 (95\% CI 0.04-0.31).

No statistically significant difference in recurrent patellofemoral instability rates between MPFL reconstruction techniques and other soft tissue realignment techniques were found (n.s.). There was a large variation in treatment effects over different settings, including what effect is to be expected in future patients.

Conclusion This systematic review and meta-analysis found that recurrent patellofemoral instability rates after MPFL reconstruction techniques are in the range of instability rates after other soft tissue realignment techniques. The clinical relevance of this study is that it provides clinicians with the best currently available evidence on recurrent patellofemoral instability rates after surgical treatment for patellofemoral instability in skeletally immature patients.
\end{abstract}

Level of evidence IV.

Keywords Patellar instability $\cdot$ Recurrent patellar dislocation $\cdot$ MPFL $\cdot$ Medial patellofemoral ligament reconstruction $\cdot$ Skeletally immature $\cdot$ Open physes

\section{Introduction}

Gerjon Hannink

gerjon.hannink@radboudumc.nl

1 Department of Orthopedics, Radboud University Medical Center, Nijmegen, The Netherlands

2 Department of Operating Rooms, Radboud University Medical Center, PO Box 9101, 6500 HB Nijmegen, The Netherlands
Patellofemoral dislocation or subluxation is a common knee injury in children and young adolescents. The overall annual incidence of patellar dislocation has been estimated to be 23.2 per 100,000 , with a highest annual incidence among adolescents aged 14 to 18 years of 147.7 per 100,000 [33]. However, as most epidemiological studies focus on the adult population, the exact numbers for skeletally immature 
patients with patellofemoral instability are still unknown [36]. The risk of recurrent instability reported in literature varies widely, ranging from 11 to $60 \%$ after primary dislocation [17, 21, 26, 32].

The pathomechanism of patellofemoral instability is complex and often multifactorial. The osseous anatomy of the entire femur, in both torsion and trochlea shape, is often abnormal and the rotation of the tibia and the ligamentous stability (i.e. laxity) of the knee have been reported to be important predisposing factors to develop patellofemoral instability or pain $[26,31,37,39]$.

After first time dislocation, conservative treatment is indicated, whereas surgery is the treatment of first choice in case of recurrence [29].

In adults, the main surgical goal is to restore the bony mismatch in the knee, for instance, by performing a tibial tubercle transfer or trochleoplasty. There is still much controversy in the current literature as to what extend and degree of bone pathology requires correction in addition to a MPFL reconstruction [29].

However, these bony surgical procedures are generally not indicated in skeletally immature patients due to the risk to damage an open growth plate, and the subsequent development of bony deformities. Nelitz et al. [23] recently showed that for selected adolescent patients with high-grade trochlear dysplasia, trochleoplasty can be safely performed up to 2 years before the projected end of growth. However, so far, soft tissue (balancing) or realignment techniques are the generally preferred operative options for skeletally immature patients [5, 34].

In the past century many realignment techniques, such as the (Roux-) Goldthwait, the Galeazzi semitendinosus tenodesis, the lateral retinaculum release, the medial retinaculum reefing/imbrication or any combined procedures have been described $[2-4,7,11,13,16,27,31,36]$. The success rates of these techniques vary widely, and none of these techniques has been shown to be superior to the other. In the last decade, several studies have shown the importance of the medial patellofemoral ligament (MPFL) as a medial restraint against lateral patellar displacement in early knee flexion, and several promising MPFL reconstruction techniques, with different grafts and/or fixation points, have been described in skeletally immature patients $[1,8,15,17,21$, 22, 27, 42].

The purpose of this systematic review and meta-analysis was to identify all available evidence on recurrent patellofemoral instability rates after MPFL reconstruction techniques and other soft tissue realignment techniques in skeletally immature patients. The results of this study will provide clinicians with the best currently available evidence on recurrent patellofemoral instability rates after surgical treatment for patellofemoral instability in skeletally immature patients, can be helpful in the process of deciding whether or not to perform such a procedure, and can be used to better inform patients about the advantages and disadvantages of different procedures.

\section{Materials and methods}

This systematic review investigates recurrent patellofemoral instability rates after MPFL reconstruction techniques and other soft tissue realignment techniques in skeletally immature patients. The inclusion criteria and method of analysis were specified in advance and documented in a protocol (PROSPERO CRD42017069706) and the study is reported according to PRISMA guidelines [20].

\section{Search strategy and selection}

Pubmed, Embase, Web of Science, and The Cochrane Library were searched (last search performed May 8, 2019) for articles concerning randomized controlled trials (RCTs), quasi-randomized trials and all observational studies. The search strategy, composed of three elements (patella, instability, and skeletally immature), was developed in collaboration with information specialists from the medical library of the Radboud university medical center Nijmegen, the Netherlands. The detailed search strategy is provided in Appendix 1.

Reference lists of the selected relevant (review) papers were screened for potentially missed papers, and no restrictions in publication date were imposed. Only articles in English, German, French and Dutch were selected. Search results were imported in EROS (Early Review Organizing Software, developed by Institute of Clinical Effectiveness and Health Policy, Buenos Aires, Argentina) to remove duplicates, and randomly allocate references to two independent reviewers responsible for screening, selection and data extraction (OW, SvdG). Discrepancies were resolved by discussion and if necessary a third reviewer was consulted (GH).

Initially, during the screening phase, primary studies evaluating any treatment for recurrent patellofemoral instability in skeletally immature patients were selected based on their title and abstract only. Review articles, letters, conference abstracts were excluded. In addition, articles with congenital (syndromic) or primary/acute patellar instability were also excluded. In the event that there was insufficient information to make a valid judgment, the whole publication was evaluated. Full-text copies of all publications eligible for inclusion were subsequently assessed and included when they met our prespecified inclusion criteria: (1) randomized controlled trials (RCTs), quasirandomized trial, or other observational study design; (2) skeletally immature patients [defined as human individuals with open physes (radiological) or age $\leq 12$ years (girls) or $\leq 14$ years 
(boys)]; (3) description of (semi-)quantitative outcome measures related to recurrent patellofemoral instability (defined as repeated dislocation or subluxation of the patella).

\section{Data extraction}

Next to bibliographic details, data on study design, number of patients, number of knees, type of intervention, and outcome measures were extracted. Attempts were made to obtain original data by contacting authors if results were presented incomplete or graphically only. If not otherwise possible, graphically presented data were converted to numerical data using digital ruler software (Plot Digitizer, University of South Alabama, USA).

\section{Risk of bias}

The quality of the included studies was assessed using the risk of bias in non-randomized studies of interventions (ROBINS-I) assessment tool by two reviewers (OW, SvdG) independently. The ROBINS-I tool uses the Cochrane-approved risk of bias approach and focuses on risk of bias due to the counterfactual and consequently articulates limitations in the assessed studies [38].

\section{Statistical analysis}

Statistical analyses were performed using $\mathrm{R}$ version 3.6.0 ( $\mathrm{R}$ Foundation for Statistical Computing, Vienna, Austria) with package 'meta'. Whenever three or more studies per surgical technique (MPFL reconstruction or other soft tissue realignment techniques) reported on recurrent patellar instability, we included these studies in our meta-analysis. Studies with $\leq 3$ patients were considered case reports and not included in the meta-analysis. Despite anticipated heterogeneity, the individual study proportions were pooled. Pooled estimates of proportions with their corresponding $95 \%$ confidence intervals (CIs) were calculated using Freeman-Tukey double arcsine transformation within a random effects model framework. Heterogeneity of combined study results was assessed by $I^{2}$, and its connected chi-square test for heterogeneity were calculated. Restricted maximum likelihood was used to estimate the variance in heterogeneity. 95\% prediction intervals (PIs) were calculated to present the expected range of true effects in similar studies [12]. Publication bias was addressed by means of a funnel plot, if at least 15 studies could be included [35].

\section{Results}

The search strategy retrieved 1433 records. The subsequent selection procedure resulted in 21 eligible articles. A flow chart of the study selection process is presented in Fig. 1.
Of the 21 eligible articles (448 knees in 389 patients), 10 studies reported on MPFL reconstruction techniques using different grafts and fixation techniques and 11 reported on other soft tissue realignment procedures. In total, 62 of the 448 (13.8\%) treated knees showed recurrent patellofemoral instability during follow-up. All 21 studies reported on different surgical techniques or combinations of surgical techniques. There was a large variation in reported recurrent instability rates, varying between 0 and $38 \%$ for MPFL reconstruction techniques and between 0 and $82 \%$ for other soft tissue realignment techniques. The characteristics of all included studies are summarized in Table 1. Mean follow-up ranged between 17.7 months and 7.4 years and between 12 months and 13.5 years for MPFL reconstruction techniques and other soft tissue realignment techniques, respectively.

\section{Risk of bias and quality of reporting}

The results of the quality assessment of all included studies are presented in Table 2. There was a considerable risk of bias in most of the included studies and the methodological quality was rated "serious" to "critical". None of the included articles were randomized nor blinded.

\section{Results of studies included in the meta-analysis}

Ten studies reporting on MPFL reconstruction techniques $[1,8,15,17,19,21,22,28,40,42]$, and nine studies reporting on other soft tissue realignment techniques [2-4, 7, 11, $16,18,27,31]$ were included in the meta-analysis (Fig. 1).

The overall pooled recurrent patellofemoral instability rate was estimated to be 0.08 (95\% CI 0.02-0.16) (Fig. 2). For MPFL reconstruction techniques, the pooled recurrent patellofemoral instability rate was estimated to be 0.02 (95\% CI 0.00-0.09) (Fig. 2). For the other soft tissue realignment techniques, the pooled rate was estimated to be 0.15 (95\% CI 0.04-0.31) (Fig. 2). The 95\% PIs reflect the variation in treatment effects over different settings, including what effect is to be expected in future patients, such as the patients that a clinician is interested to treat. The PIs reflect the large heterogeneity in both the MPFL reconstruction techniques [95\% PI, 0.00-0.27 (heterogeneity: $I^{2}=60 \%$; $p<0.01)]$ and the other soft tissue realignment techniques [95\% PI, 0.00-0.77 (heterogeneity: $I^{2}=89 \% ; p \leq 0.01$ )]. No statistically significant difference in recurrent patellofemoral instability rates between MPFL reconstruction techniques and other soft tissue realignment techniques were found $\left(\chi^{2}=3.04\right.$; n.s. $)$. 


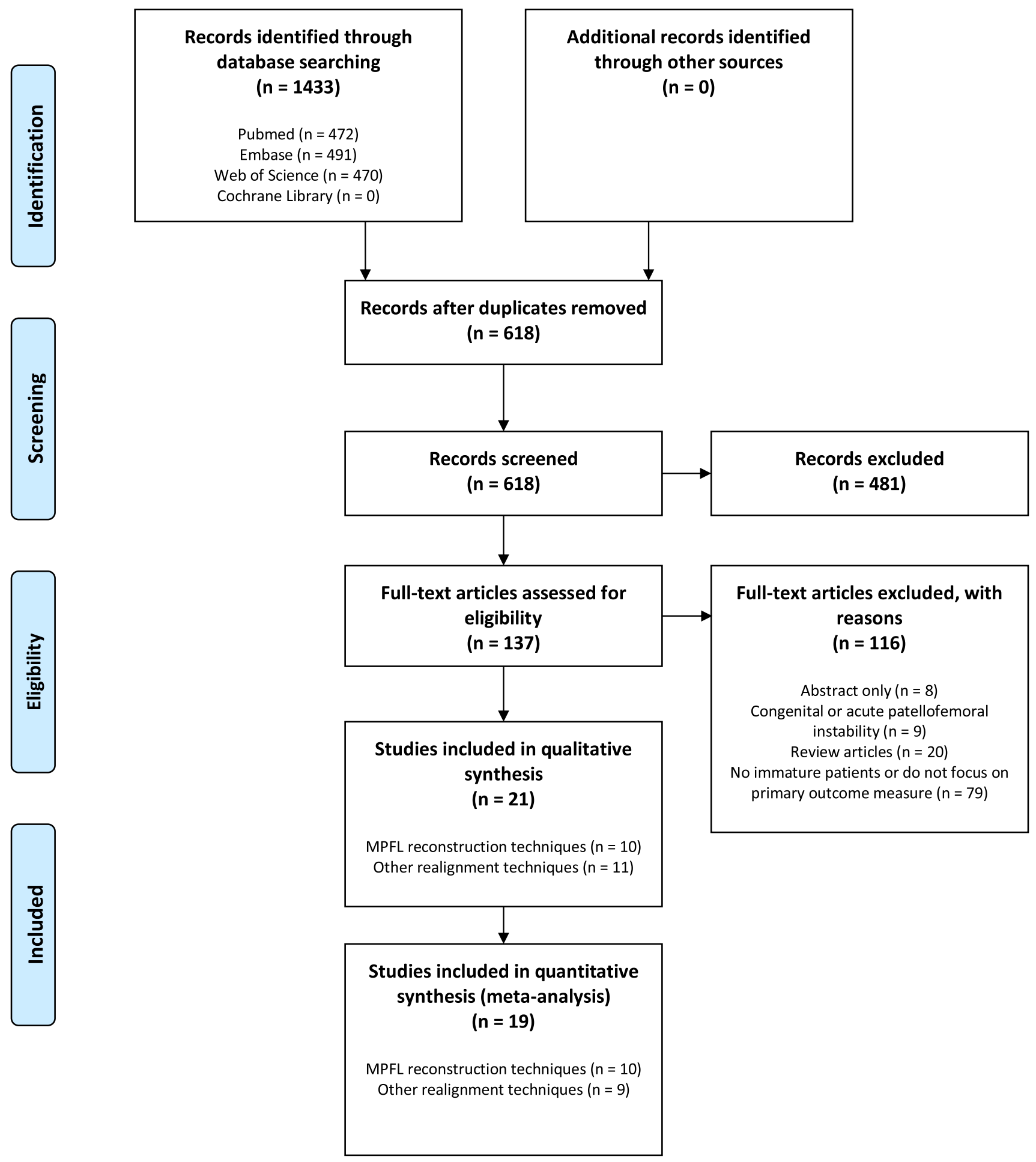

Fig. 1 PRISMA flowchart of search results

\section{Results of studies not included in the meta-analysis}

All studies on MPFL reconstruction techniques were included in the meta-analysis. Sugimoto et al. [36] and
Joo et al. [13] were not included in the meta-analysis for the other soft tissue realignment techniques as these were considered case-reports. Sugimoto et al. [36] performed a Roux-Goldthwait procedure and a lateral release combined with a medial capsular reefing on two patients, and Joo 


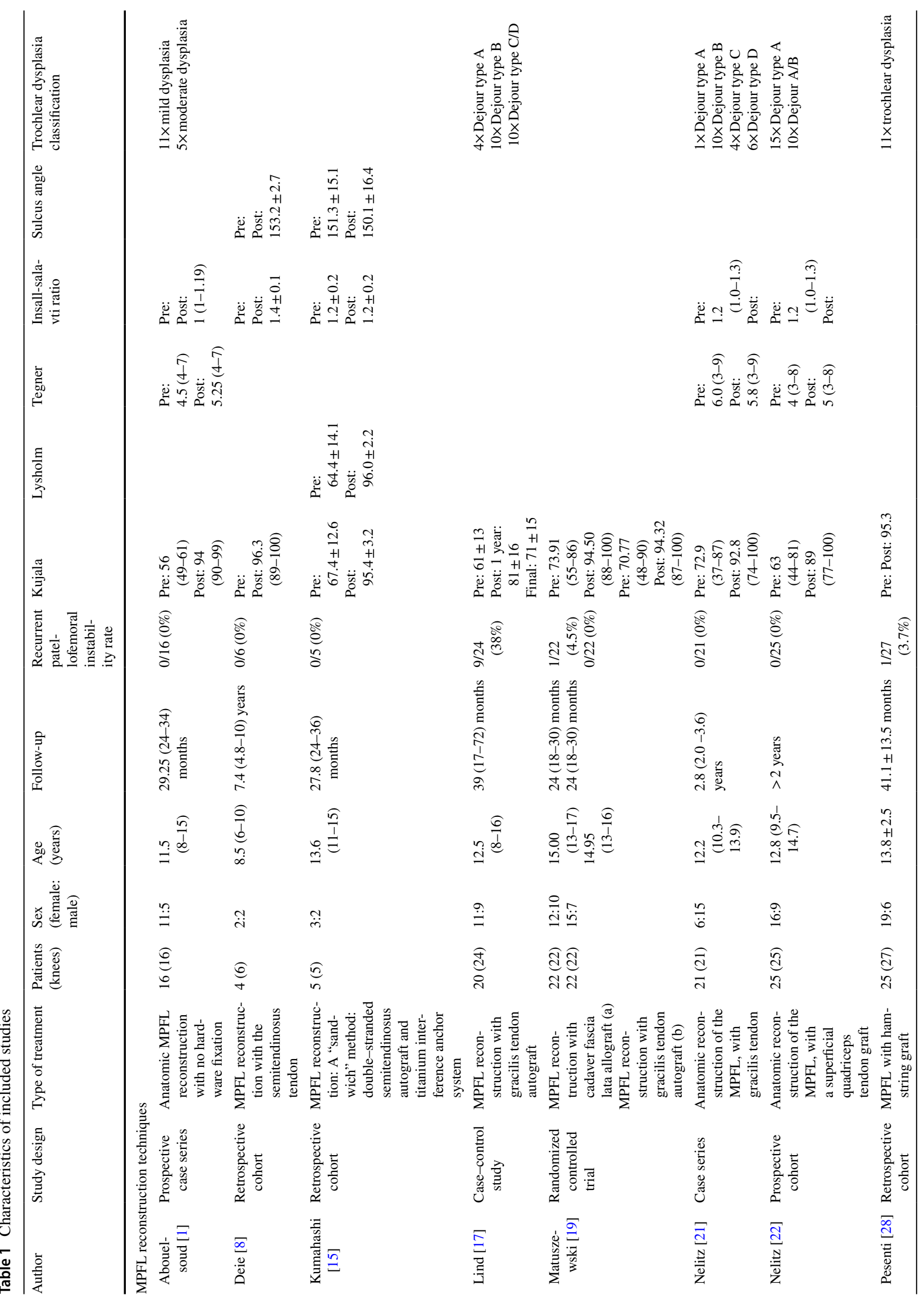




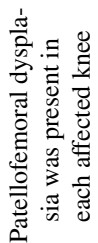

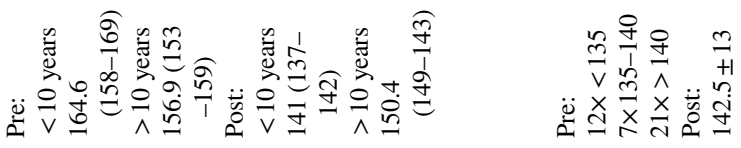

$\ddot{D} \ddot{\ddot{\theta}}$

$\ddot{g} \ddot{\ddot{\theta}}$

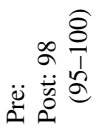

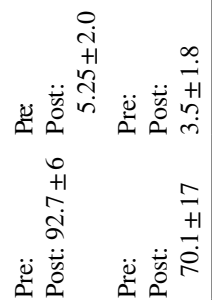

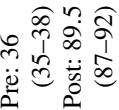

$\ddot{0} \ddot{\ddot{0}}$

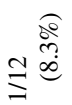

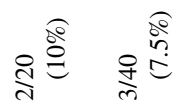

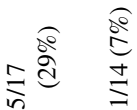

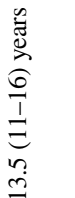

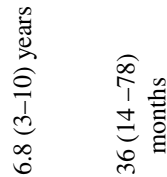

ָे

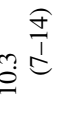

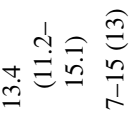

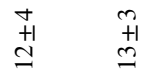

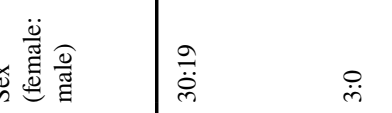

$\stackrel{\infty}{=} \quad \check{\square}$

व్d

m.

竞

孚孚

$\underset{\infty}{\widehat{C}}$

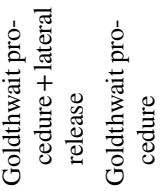

$\underset{I}{\stackrel{\Im}{\Xi}}$

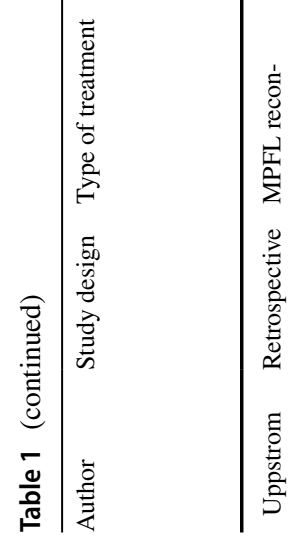

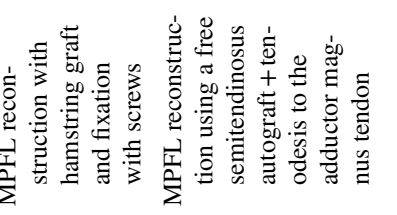

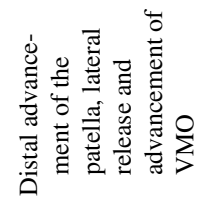

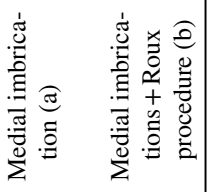

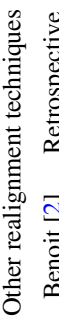

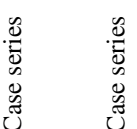

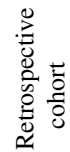

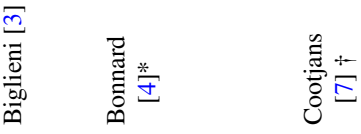




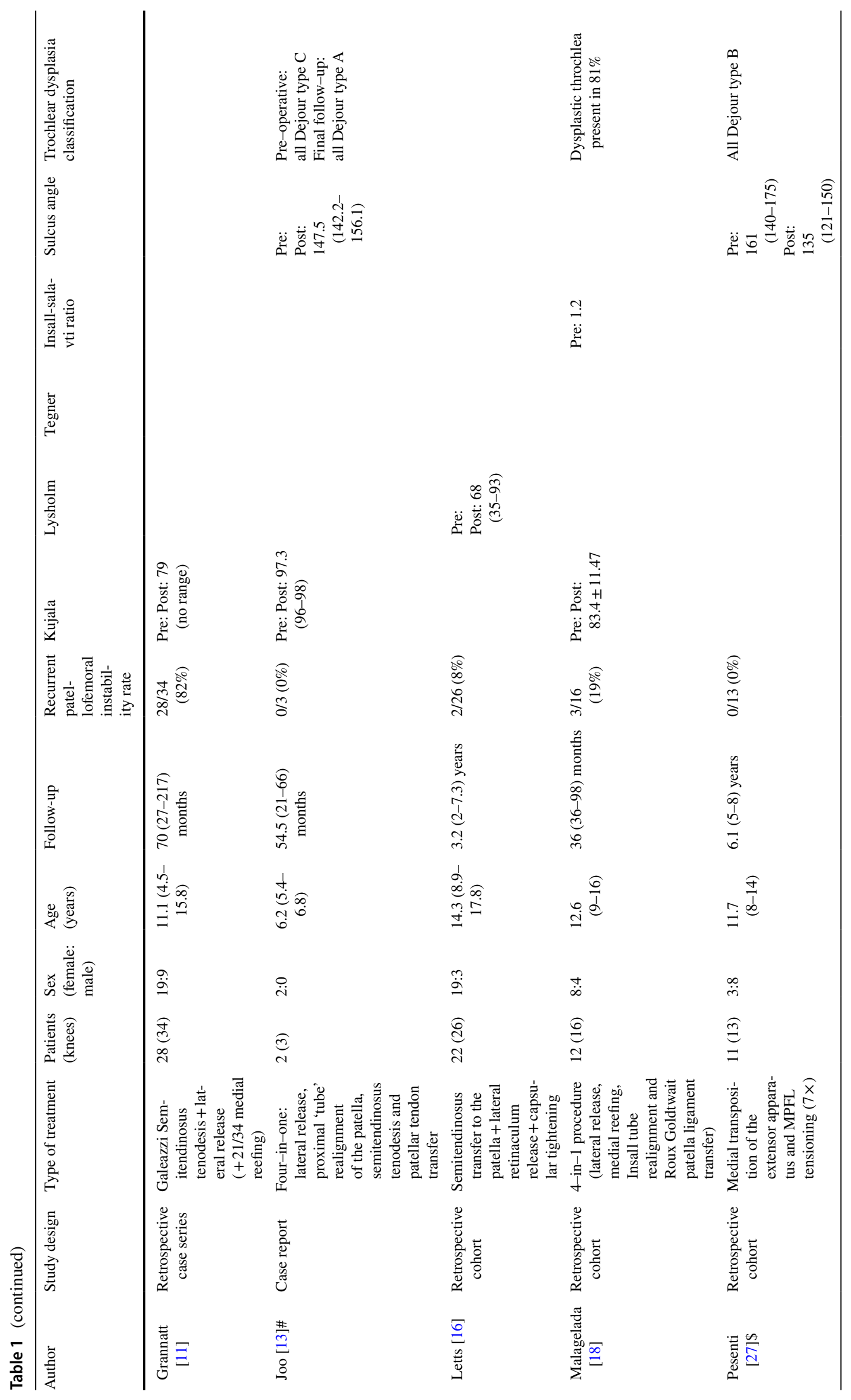




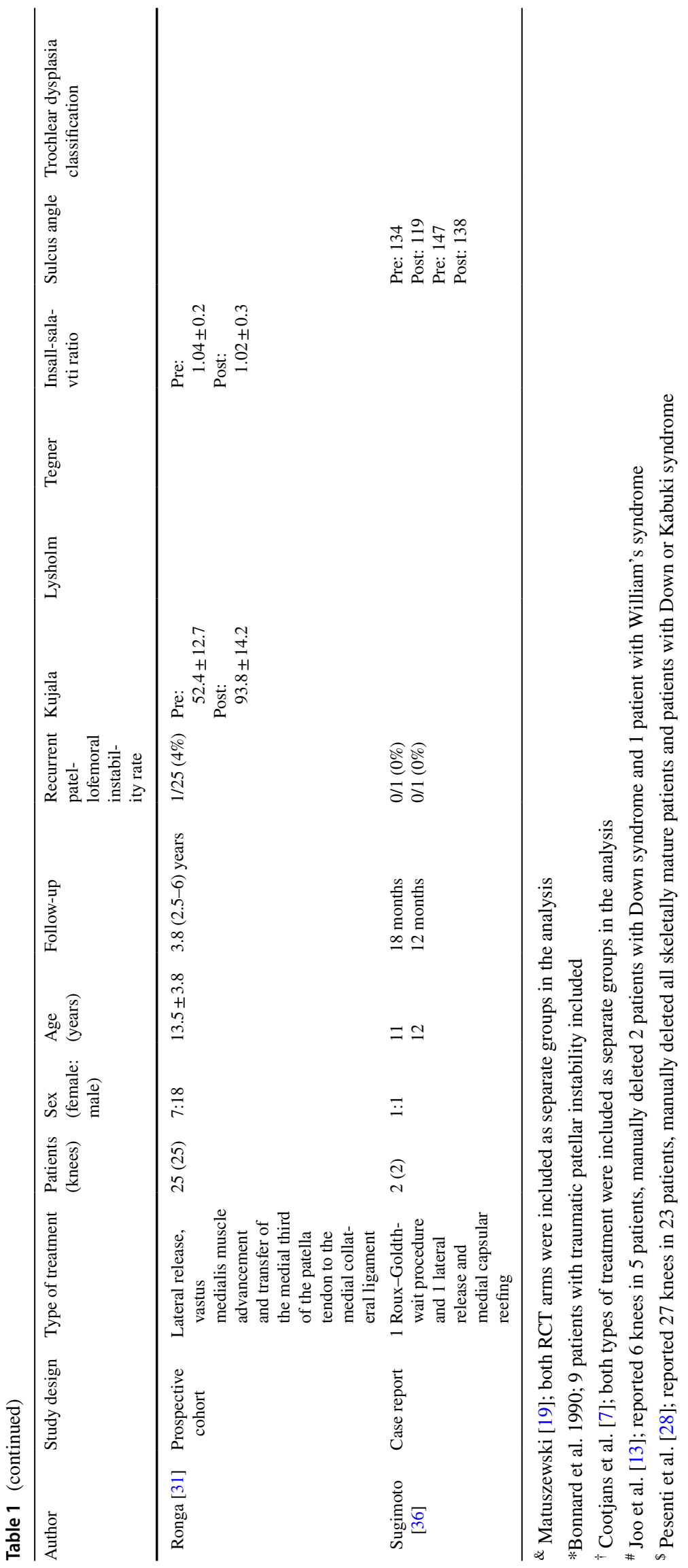


Table 2 ROBINS-I Risk of bias assessment

$\begin{array}{llllllll}\text { Domain 1: } & \text { Domain 2: } & \text { Domain 3: } & \text { Domain 4: } & \text { Domain } \\ \text { confound- } & \text { selection of } \\ \text { ing } & \text { participants } & \begin{array}{l}\text { Domain 6: } \\ \text { of intervention }\end{array} & \begin{array}{l}\text { deviation from } \\ \text { interventions }\end{array} & \begin{array}{l}\text { 5: missing } \\ \text { data }\end{array} & \begin{array}{l}\text { measurement } \\ \text { of outcomes }\end{array} & \begin{array}{l}\text { Selection } \\ \text { of reported } \\ \text { results }\end{array} & \begin{array}{l}\text { ROBINS-I } \\ \text { overall }\end{array} \\ & & & & & \end{array}$

\begin{tabular}{|c|c|c|c|c|c|c|c|c|}
\hline \multicolumn{9}{|c|}{ MPFL reconstruction techniques } \\
\hline $\begin{array}{l}\text { Abouelsoud } \\
\text { [1] }\end{array}$ & ${ }^{\mathrm{F}} 0$ & 3 & $2-3$ & ${ }^{\mathrm{F}} 0$ & 3 & 3 & ${ }^{\mathrm{F}} 0$ & Serious \\
\hline Deie $[8]$ & ${ }^{\mathrm{F}} 0$ & 3 & 3 & ${ }^{\mathrm{F}} 0$ & 2 & 3 & ${ }^{\mathrm{F}} 0$ & Serious \\
\hline $\begin{array}{l}\text { Kumahashi } \\
\text { [15] }\end{array}$ & ${ }^{\mathrm{T}} 0$ & 3 & 2 & $2-3$ & 2 & 3 & ${ }^{\mathrm{F}} 0$ & $\begin{array}{l}\text { Moderate-seri- } \\
\text { ous }\end{array}$ \\
\hline Lind [17] & 4 & 3 & 3 & ${ }^{\mathrm{F}} 0$ & 3 & 3 & ${ }^{\mathrm{F}} 0$ & Serious \\
\hline $\begin{array}{l}\text { Matuszewski } \\
\text { [19] }\end{array}$ & 1 & 1 & 1 & 1 & 1 & 1 & 1 & Low \\
\hline Nelitz [21] & ${ }^{\mathrm{F}} 0$ & 3 & $2-3$ & ${ }^{\mathrm{F}} 0$ & 2 & 3 & ${ }^{\mathrm{F}} 0$ & Serious \\
\hline Nelitz [22] & ${ }^{\mathrm{F}} 0$ & $2-3$ & $2-3$ & $2-3$ & 2 & 3 & 2 & $\begin{array}{l}\text { Moderate-seri- } \\
\text { ous }\end{array}$ \\
\hline Pesenti [28] & ${ }^{\mathrm{F}} 0$ & 3 & 3 & 3 & 3 & 3 & ${ }^{\mathrm{F}} 0$ & Serious \\
\hline $\begin{array}{l}\text { Uppstrom } \\
{[40]}\end{array}$ & Ғ0 & 3 & 2 & 2 & 2 & 2 & 2 & $\begin{array}{l}\text { Moderate-seri- } \\
\text { ous }\end{array}$ \\
\hline Yercan [42] & ${ }^{\mathrm{F}} 0$ & $3-4$ & 4 & ${ }^{\mathrm{F}} 0$ & 3 & ${ }^{\mathrm{F}} 0$ & ${ }^{\mathrm{F}} 0$ & Serious-critical \\
\hline \multicolumn{9}{|c|}{ Other realignment techniques } \\
\hline Benoit [2] & ${ }^{\mathrm{F}} 0$ & 3 & $2-3$ & ${ }^{\mathrm{F}} 0$ & 2 & 3 & $2-3$ & $\begin{array}{l}\text { Moderate-seri- } \\
\text { ous }\end{array}$ \\
\hline Biglieni [3] & ${ }^{\mathrm{F}} 0$ & 4 & 4 & ${ }^{\mathrm{F}} 0$ & $3-4$ & 3 & ${ }^{\mathrm{F}} 0$ & Serious-critical \\
\hline Bonnard [4] & ${ }^{\mathrm{F}} 0$ & 3 & 3 & ${ }^{\mathrm{F}} 0$ & 3 & 3 & ${ }^{\mathrm{F}} 0$ & Serious \\
\hline Cootjans [7] & ${ }^{\mathrm{F}} 0$ & 4 & 4 & ${ }^{\mathrm{F}} 0$ & 4 & 4 & ${ }^{\mathrm{F}} 0$ & Serious-critical \\
\hline Grannatt [11] & $2-3$ & 3 & 3 & $3-4$ & 3 & 3 & 3 & Serious \\
\hline Joo [13] & ${ }^{\mathrm{F}} 0$ & ${ }^{\mathrm{F}} 0$ & 4 & 3 & 3 & ${ }^{\mathrm{F}} 0$ & $\mathrm{~F}_{0}$ & Serious \\
\hline Letts [16] & ${ }^{\mathrm{F}} 0$ & 3 & 3 & $2-3$ & $2-3$ & 3 & 3 & Serious \\
\hline $\begin{array}{l}\text { Malagelada } \\
{[18]}\end{array}$ & Ғ0 & 3 & 2 & 2 & 2 & 2 & 2 & $\begin{array}{l}\text { Moderate-seri- } \\
\text { ous }\end{array}$ \\
\hline Pesenti [27] & ${ }^{\mathrm{F}} 0$ & $3-4$ & $3-4$ & $2-3$ & 2 & 3 & ${ }^{\mathrm{F}} 0$ & Serious \\
\hline Ronga [31] & ${ }^{\mathrm{F}} 0$ & 3 & $2-3$ & ${ }^{\mathrm{F}} 0$ & 3 & 3 & ${ }^{\mathrm{F}} 0$ & Serious \\
\hline $\begin{array}{l}\text { Sugimoto } \\
{[36]}\end{array}$ & ${ }^{\mathrm{F}} 0$ & 4 & ${ }^{\mathrm{F}} 0$ & 4 & ${ }^{\mathrm{F}} 0$ & 4 & 4 & Critical \\
\hline
\end{tabular}

Risk of bias assessment: 0 No information; 1 low; 2 moderate; 3 serious; 4 critical

Ғ0 (no information) was assessed as equivalent to "Serious" (3)

et al. [13] performed a four-in-one procedure: a lateral release, proximal 'tube' realignment of the patella, semitendinosus tenodesis and patellar tendon transfer on two patients. Both studies reported 0\% recurrent patellofemoral instability rates.

\section{Publication bias}

Due to the low number of studies that were included in the meta-analyses the possible presence of publication bias could not reliably be assessed.

\section{Discussion}

The most important finding of this systematic review and meta-analysis was that recurrent patellofemoral instability rates using MPFL reconstruction techniques were in the range of instability rates after other soft tissue realignment techniques. There was a large variation in both surgical techniques and reported recurrent instability rates, varying between 0 and $38 \%$ for MPFL reconstruction techniques and between 0 and $82 \%$ for other soft tissue realignment techniques.

In the MPFL reconstruction techniques, 9 out of 10 studies reported low post-operative recurrent patellofemoral instability rates. Only Lind et al. [17] reported a 38\% (9/24 


\section{MPFL reconstruction techniques}

\begin{tabular}{lrr} 
Abouelsoud, 2015' & 0 & 16 \\
Deie, 2003 & 0 & 6 \\
Kumahashi, 2012 & 0 & 5 \\
Lind, 2016 $^{17}$ & 9 & 24 \\
Matuszewski, 2018a & 1 & 22 \\
Matuszewski, 2018b & 0 & 22 \\
Nelitz, 2013 & 0 & 21 \\
Nelitz, 2018 & 0 & 25 \\
Pesenti, 2018 & 1 & 27 \\
Uppstrom, 2019 & 5 & 54 \\
Yercan, 2011 & 0 & 4 \\
Random effects model & \multicolumn{2}{c}{226} \\
Heterogeneity: $\left.\right|^{2}=60 \%, T^{2}=0.0179, X_{2}^{2}=25.13(p<0.01)$
\end{tabular}

Other soft tissue realignment techniques Benoit, $2007^{2}$

Biglieni, $2011^{3}$

Bonnard, $1990^{4}$

Cootjans, 2013a ${ }^{7}$

Cootjans, 2013b

Grannatt, 2012 11

Letts, $1999^{16}$

Malagelada, $2018^{18}$

Pesenti, $2017^{27}$

Ronga, 2009 ${ }^{31}$

Random effects model

年 $1^{2}=89 \%, \tau^{2}=0.0678, x^{2}=82.55(p<0.01)$

\section{Random effects model} Prediction interval

Heterogeneity: $I^{2}=84 \%, \tau^{2}=0.0477, x_{20}^{2}=126.17(p<0.01)$ Residual heterogeneity: $I^{2}=82 \%, x_{19}^{2}=107.68(p<0.01)$ Test for subgroup differences: $\quad x_{1}^{2}=3.04, d f=1 \quad$ (n.s.)

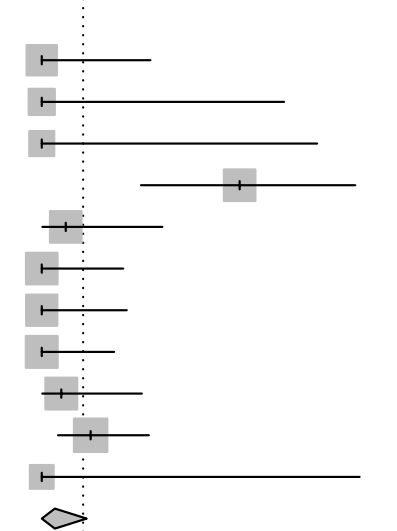

$0.00 \quad[0.00 ; 0.21]$

$4.7 \%$

$0.00 \quad[0.00 ; 0.46]$

$3.5 \%$

$0.00 \quad[0.00 ; 0.52]$

$3.2 \%$

$0.38 \quad[0.19 ; 0.59]$

$5.1 \%$

$0.05 \quad[0.00 ; 0.23]$

$5.1 \%$

$0.00 \quad[0.00 ; 0.15]$

$5.1 \%$

$0.00 \quad[0.00 ; 0.16] \quad 5.0 \%$

$0.00 \quad[0.00 ; 0.14] \quad 5.2 \%$

$0.04 \quad[0.00 ; 0.19] \quad 5.2 \%$

$0.09 \quad[0.03 ; 0.20] \quad 5.7 \%$

$0.00 \quad[0.00 ; 0.60] \quad 2.9 \%$

$0.02[0.00 ; 0.09] \quad 50.7 \%$
Fig. 2 Forest plots of the included studies using the MPFL reconstruction techniques and other soft tissue realignment techniques. Forest plots display the proportion of complications, 95\% confidence interval and the relative weight of the individual studies. The diamond indicates the pooled estimate and its $95 \%$ confidence interval. The red bar indicates the $95 \%$ prediction interval. Prediction intervals illustrate which range of true effects expected to occur in similar studies in future settings. Matuszewski et al. [19] reported a randomized

knees) recurrent patellofemoral instability rate at final follow-up. They reported on patients with various degrees of patellofemoral dysplasia, which may explain the high recurrent patellofemoral instability rate. However, they could not find an association between the high degree of trochlea dysplasia (grade C and D) and the redislocation rates [17]. Abouelsoud et al. [1] reported no recurrent patellofemoral instability after MPFL reconstruction, but five cases could be described as infrequent subluxation episodes. Patients with severe trochlear dysplasia were excluded in their study. controlled trial (RCT) comparing two different MPFL reconstruction techniques using a fascia lata allograft, and $\mathbf{b}$ gracilis tendon autograft. Both RCT arms were included as separate groups in the analysis. Cootjans et al. [7] reported a retrospective cohort study consisting of two cohorts using a medial imbrication alone, and $\mathbf{b}$ medial imbrication combined with a Roux procedure. Both cohorts were included as separate groups in the analysis

In the other soft tissue realignment techniques, 8 out of 11 studies reported post-operative recurrent instability rates less than or equal to $10 \%$. Two studies reported rates higher than 20\%. Cootjans et al. [7] reported a recurrent instability rate of $29 \%$, while Grannatt et al. [11], reported an $82 \%$ rate at final follow-up. An explanation for the poor results in Grannatt et al. [11] could be the long duration of follow-up. Patients had a minimum follow-up of 2-year with a mean follow-up of 5.8 years (range 27-217 months). They concluded that the Galeazzi procedure may be associated with higher 
rates of recurrent instability and more debilitated knee function than previously appreciated. Cootjans et al. [7] reported a very low response rate on the questionnaire and analyzed and reported the data based on the available questionnaires.

Despite the more anatomical nature of a MPFL reconstruction, in the present study no clear advantage of MPFL reconstruction techniques over other soft tissue realignment techniques was found as the confidence intervals were overlapping. One of the most important reasons for MPFL reconstruction failure in young patients is severe trochlear dysplasia [24]. Generally, this is not addressed until patients have closed physes. Since younger patients have often more severe dysplasia or rotational deformities, this might explain the similar recurrence rates in both groups. Despite severe trochlear dysplasia or increased femoral anteversion, technical errors (e.g. non-anatomic bone tunnels or overtensioning of the graft) are also a common cause for MPFL reconstruction failure [25]. An MPFL reconstruction remains a challenging procedure in young patients, particularly in those with additional bony deformities with attribute to patellar instability, and should, therefore, be performed by experienced surgeons.

Some limitations of this study have to be discussed. First, the definition of recurrent patellofemoral instability, that is redislocation or subluxation, is arguable and might differ between clinicians and/or patients. In addition, relying on patient reported recurrent patellofemoral instability may result in not all occurrences being reported. Second, there is no clear consensus on indication for the use an MPFL reconstruction techniques or other soft tissue realignment techniques, which hampers a comparison between studies and/or techniques. The presented recurrent patellofemoral instability rates for different techniques should be interpreted in the context of the individual studies that have been published, including exact indication for surgery, duration and severity of symptoms, and patient factors. Predisposing factors, such as increased Q-angle and TT-TG in combination with all the limitations of soft tissue procedures could also explain a high recurrent patellofemoral instability rate, but these are unknown for all individual patients included in the studies. Third, almost all studies were retrospective or prospective case series and publication bias may be present since "negative" results of case series of surgical procedures are less likely to be submitted for publication. None of the studies were randomized nor blinded and there was a considerable risk of bias in most of the included studies.

Skeletally immature patients have the unique advantage that their bones are capable of remodeling after injury or a surgical intervention. Sugimoto et al. [36] described a decrease in sulcus angle after surgery, suggesting the femoral trochlea was deepened and remodelled due to a more centralized patella. Joo et al. [13] concluded an improvement in development of the femoral trochlea after surgery. In contrast, Rajdev et al. [30] showed no remodeling of the femoral trochlea after patellar stabilization. However, in that study the mean age was 14.7 years and therefore most of the patients were after their growth spurt. In the same study, the age of 10 years is indicated as an important age after which trochlear remodeling is limited. It can be assumed that with a skeletal age of 12 years for girls and 14 years for boys, the main growth spurt is over and there is only residual growth after that and no clear remodeling is to be expected [23]. This conclusion is also supported by Fu et al. [9], who showed trochlear remodeling in a patient population ranging from 7-11 years of age. Therefore, the relation between soft tissue realignment and remodeling seems to be clearly related to the start of the growth spurt of a patient. Evidence is still very limited due to lack of information in most studies. Nevertheless, trochlear remodeling due to patella realignment surgery is a topic which needs to be addressed more in detail, since it has not been well described in the pediatric orthopedic literature yet.

Many different surgical techniques on skeletally mature patients have been reported. These surgical techniques may be used on skeletally immature patients, however, should be possibly modified and further studied before use in this young patient population $[6,10,14,41]$.

The clinical relevance of this study is that it provides clinicians with the best currently available evidence on recurrent patellofemoral instability rates after surgical treatment for patellofemoral instability in skeletally immature patients. This can be helpful in the process of deciding whether or not to perform such a procedure, and can be used to better inform patients about the advantages and disadvantages of different procedures.

\section{Conclusion}

This systematic review and meta-analysis found that recurrent patellofemoral instability rates after MPFL reconstruction techniques are in the range of instability rates after other soft tissue realignment techniques.

Author contributions $\mathrm{OW}, \mathrm{GH}$, and SvdG designed the study and drafted the research protocol. OW and SvdG performed the literature search, selection, data extraction and drafted the manuscript. OE and GH developed the search strategy and performed the data analysis. All authors contributed in the interpretation of the data. All authors read and approved the final version of the manuscript. GH is corresponding author.

Funding No external source of funding was used.

\section{Compliance with ethical standards}

Conflict of interest The authors declare that they have no conflict of interest. 
Ethical approval No ethical approval was obtained because this study was a systematic review with meta-analysis using anonymized data from other published cohort studies.

Informed consent For this type of study informed consent is not required.

Availability of data and materials The datasets generated and/or analyzed during the current study are not publicly available but are available from the corresponding author $(\mathrm{GH})$ on reasonable request.

Open Access This article is distributed under the terms of the Creative Commons Attribution 4.0 International License (http://creativeco mmons.org/licenses/by/4.0/), which permits unrestricted use, distribution, and reproduction in any medium, provided you give appropriate credit to the original author(s) and the source, provide a link to the Creative Commons license, and indicate if changes were made.

\section{References}

1. Abouelsoud MM, Abdelhady A, Elshazly O (2015) Anatomic physeal-sparing technique for medial patellofemoral ligament reconstruction in skeletally immature patients with ligamentous laxity. Eur J Orthop Surg Traumatol 25:921-926

2. Benoit B, Laflamme GY, Laflamme GH, Rouleau D, Delisle J, Morin B (2007) Long-term outcome of surgically-treated habitual patellar dislocation in children with coexistent patella alta: minimum follow-up of 11 years. J Bone Joint Surg Br 89:1172-1177

3. Biglieni L, Fiore M, Coviello M, Felli L (2011) Patellar instability: combined treatment with Goldthwait technique and arthroscopic lateral release. Musculoskelet Surg 95:95-99

4. Bonnard C, Nocquet P, Sollogoub I, Glorion B (1990) Patellar instability in children. Result of transposition of the medial third of patellar tendon. Rev Chir Orthop Reparatrice Appar Mot 76:473-479

5. Camathias C, Rutz E, Gotze M, Brunner R, Vavken P, Gaston MS (2014) Poor outcome at 7.5 years after Stanisavljevic quadriceps transposition for patello-femoral instability. Arch Orthop Trauma Surg 134:473-478

6. Coons DA, Barber FA (2006) Thermal medial retinaculum shrinkage and lateral release for the treatment of recurrent patellar instability. Arthroscopy 22:166-171

7. Cootjans K, Dujardin J, Vandenneucker H, Bellemans J (2013) A surgical algorithm for the treatment of recurrent patellar dislocation. Results at 5 year follow-up. Acta Orthop Belg 79:318-325

8. Deie M, Ochi M, Sumen Y, Yasumoto M, Kobayashi K, Kimura $H$ (2003) Reconstruction of the medial patellofemoral ligament for the treatment of habitual or recurrent dislocation of the patella in children. J Bone Joint Surg Br 85:887-890

9. Fu K, Duan G, Liu C, Niu J, Wang F (2018) Changes in femoral trochlear morphology following surgical correction of recurrent patellar dislocation associated with trochlear dysplasia in children. Bone Joint J 100:811-821

10. Goyal D (2013) Medial patellofemoral ligament reconstruction: the superficial quad technique. Am J Sports Med 41:1022-1029

11. Grannatt K, Heyworth BE, Ogunwole O, Micheli LJ, Kocher MS (2012) Galeazzi semitendinosus tenodesis for patellofemoral instability in skeletally immature patients. J Pediatr Orthop $32: 621-625$

12. IntHout J, Ioannidis JP, Rovers MM, Goeman JJ (2016) Plea for routinely presenting prediction intervals in meta-analysis. BMJ Open 6:e010247
13. Joo SY, Park KB, Kim BR, Park HW, Kim HW (2007) The 'four-in-one' procedure for habitual dislocation of the patella in children: early results in patients with severe generalised ligamentous laxity and aplasis of the trochlear groove. J Bone Joint Surg Br 89:1645-1649

14. Kodkani PS (2016) "Basket weave technique" for medial patellofemoral ligament reconstruction: clinical outcome of a prospective study. Indian J Orthop 50:34-42

15. Kumahashi N, Kuwata S, Tadenuma T, Kadowaki M, Uchio Y (2012) A "sandwich" method of reconstruction of the medial patellofemoral ligament using a titanium interference screw for patellar instability in skeletally immature patients. Arch Orthop Trauma Surg 132:1077-1083

16. Letts RM, Davidson D, Beaule P (1999) Semitendinosus tenodesis for repair of recurrent dislocation of the patella in children. J Pediatr Orthop 19:742-747

17. Lind M, Enderlein D, Nielsen T, Christiansen SE, Fauno P (2016) Clinical outcome after reconstruction of the medial patellofemoral ligament in paediatric patients with recurrent patella instability. Knee Surg Sports Traumatol Arthrosc 24:666-671

18. Malagelada F, Rahbek O, Sahirad C, Ramachandran M (2018) Results of operative 4-in-1 patella realignment in children with recurrent patella instability. J Orthop 15:13-17

19. Matuszewski L, Trams M, Ciszewski A, Wilczynski M, Trams E, Jakubowski P, Matuszewska A, John K (2018) Medial patellofemoral ligament reconstruction in children: a comparative randomized short-term study of fascia lata allograft and gracilis tendon autograft reconstruction. Medicine (Baltimore) 97:e13605

20. Moher D, Liberati A, Tetzlaff J, Altman DG (2009) Preferred reporting items for systematic reviews and meta-analyses: the PRISMA statement. PLoS Med 6:e1000097

21. Nelitz M, Dreyhaupt J, Reichel H, Woelfle J, Lippacher S (2013) Anatomic reconstruction of the medial patellofemoral ligament in children and adolescents with open growth plates: surgical technique and clinical outcome. Am J Sports Med 41:58-63

22. Nelitz M, Dreyhaupt J, Williams SRM (2018) Anatomic reconstruction of the medial patellofemoral ligament in children and adolescents using a pedicled quadriceps tendon graft shows favourable results at a minimum of 2-year follow-up. Knee Surg Sports Traumatol Arthrosc 26:1210-1215

23. Nelitz M, Dreyhaupt J, Williams SRM (2018) No growth disturbance after trochleoplasty for recurrent patellar dislocation in adolescents with open growth plates. Am J Sports Med 46:3209-3216

24. Nelitz M, Theile M, Dornacher D, Wolfle J, Reichel H, Lippacher S (2012) Analysis of failed surgery for patellar instability in children with open growth plates. Knee Surg Sports Traumatol Arthrose 20:822-828

25. Nelitz M, Williams RS, Lippacher S, Reichel H, Dornacher D (2014) Analysis of failure and clinical outcome after unsuccessful medial patellofemoral ligament reconstruction in young patients. Int Orthop 38:2265-2272

26. Palmu S, Kallio PE, Donell ST, Helenius I, Nietosvaara Y (2008) Acute patellar dislocation in children and adolescents: a randomized clinical trial. J Bone Joint Surg Am 90:463-470

27. Pesenti S, Blondel B, Armaganian G, Parratte S, Bollini G, Launay F, Jouve JL (2017) The lateral wedge augmentation trochleoplasty in a pediatric population: a 5-year follow-up study. J Pediatr Orthop B 26:458-464

28. Pesenti S, Ollivier M, Escudier JC, Cermolacce M, Baud A, Launay F, Jouve JL, Choufani E (2018) Medial patellofemoral ligament reconstruction in children: do osseous abnormalities matter? Int Orthop 42:1357-1362

29. Post WR, Fithian DC (2018) Patellofemoral instability: a consensus statement from the AOSSM/PFF patellofemoral instability workshop. Orthop J Sports Med 6:2325967117750352 
30. Rajdev NR, Parikh SN (2019) Femoral trochlea does not remodel after patellar stabilization in children older than 10 years of age. J Pediatr Orthop B 28:139-143

31. Ronga M, Oliva F, Longo U, Testa V, Cherubino P, Maffulli N (2011) The three-in-one procedure for recurrent dislocation of the patella in skeletally immature children and adolescents. J Orthop Traumatol 12:S138-S139

32. Sanders TL, Pareek A, Hewett TE, Stuart MJ, Dahm DL, Krych AJ (2018) High rate of recurrent patellar dislocation in skeletally immature patients: a long-term population-based study. Knee Surg Sports Traumatol Arthrosc 26:1037-1043

33. Sanders TL, Pareek A, Hewett TE, Stuart MJ, Dahm DL, Krych AJ (2018) Incidence of first-time lateral patellar dislocation: a 21-year population-based study. Sports Health 10:146-151

34. Shubin Stein BE, Ahmad CS (2007) The management of patellar instability in the skeletally immature patient. Oper Tech Orthop $17: 250-256$

35. Simmonds M (2015) Quantifying the risk of error when interpreting funnel plots. Syst Rev 4:24

36. Sugimoto D, Christino MA, Micheli LJ (2016) Effects of surgical intervention on trochlear remodeling in pediatric patients with recurrent patella dislocation cases. J Pediatr Orthop B 25:349-353

37. Tan SHS, Ibrahim MM, Lee ZJ, Chee YKM, Hui JH (2018) Patellar tracking should be taken into account when measuring radiographic parameters for recurrent patellar instability. Knee Surg Sports Traumatol Arthrosc 26:3593-3600
38. Thomson H, Craig P, Hilton-Boon M, Campbell M, Katikireddi SV (2018) Applying the ROBINS-I tool to natural experiments: an example from public health. Syst Rev 7:15

39. Tompkins MA, Rohr SR, Agel J, Arendt EA (2018) Anatomic patellar instability risk factors in primary lateral patellar dislocations do not predict injury patterns: an MRI-based study. Knee Surg Sports Traumatol Arthrosc 26:677-684

40. Uppstrom TJ, Price M, Black S, Gausden E, Haskel J, Green DW (2019) Medial patellofemoral ligament (MPFL) reconstruction technique using an epiphyseal femoral socket with fluoroscopic guidance helps avoid physeal injury in skeletally immature patients. Knee Surg Sports Traumatol Arthrosc.

41. Vavalle G, Capozzi M (2016) Isolated reconstruction of the medial patellofemoral ligament with autologous quadriceps tendon. $\mathrm{J}$ Orthop Traumatol 17:155-162

42. Yercan HS, Erkan S, Okcu G, Ozalp RT (2011) A novel technique for reconstruction of the medial patellofemoral ligament in skeletally immature patients. Arch Orthop Trauma Surg 131:1059-1065

Publisher's Note Springer Nature remains neutral with regard to jurisdictional claims in published maps and institutional affiliations. 